\title{
Pathogenesis and progression of oesophageal adenocarcinoma varies by prior diagnosis of Barrett's oesophagus
}

\author{
Michael B Cook ${ }^{*},{ }^{1}$ Jennifer Drahos ${ }^{1}$, Shannon Wood ${ }^{1}$, Lindsey Enewold ${ }^{2}$, Ruth Parsons ${ }^{3}$, Neal D Freedman ${ }^{1}$, \\ Philip R Taylor ${ }^{1}$, Winnie Ricker ${ }^{3}$ and Christian C Abnet ${ }^{1}$ \\ ${ }^{1}$ Division of Cancer Epidemiology and Genetics, National Cancer Institute, NIH, DHHS, Rockville, MD 20850, USA; ${ }^{2}$ Division of \\ Cancer Control and Population Sciences, National Cancer Institute, NIH, DHHS, Rockville, MD 20850, USA and ${ }^{3}$ Information \\ Management Services, Rockville, MD 20852, USA
}

\begin{abstract}
Background: The absolute risk of oesophageal adenocarcinoma (EA) among individuals with Barrett's oesophagus (BE) is low and a majority of EA cases are diagnosed among individuals with no prior BE diagnosis. To ensure that insights from EA case-control studies are transferable to clinical management of BE populations, we conducted a case-case study to compare the clinical presentation, medical history and survival of EA cases with and without a prior BE diagnosis in the Surveillance, Epidemiology and End Results Medicare database.
\end{abstract}

Methods: Eligible EA cases were diagnosed at age $\geqslant 68$ years during 1994-2009. There were 5271 EA cases in this study, $87 \%$ of which did not have a prior diagnosis of BE (EA-no prior BE).

Results: Multivariable case-case comparisons evidenced adverse associations of GERD, ever cigarette smoking, hypertension, dyslipidemia, weight loss, peptic ulcer and irritable bowel disease each in EA-prior BE compared with EA-no prior BE. Obesity, metabolic syndrome, impaired fasting glucose and diabetes did not differ between groups. EA-prior BE cases were diagnosed with less advanced disease, were more likely to undergo surgery and less likely to receive chemotherapy and radiotherapy, and had better overall mean survival (2.5 vs 1.4 years). This survival advantage persisted in the multivariable Cox model $(H R=0.69$, $95 \% \mathrm{Cl}: 0.60,0.78)$, despite adjustment for many factors including stage, grade and clinical interventions.

Conclusions: This study provides evidence that EA cases occurring among individuals previously diagnosed with BE are different from the large majority of EA cases that occur without a prior BE diagnosis. Regardless of whether these differences emanate from aetiology, biology and/or selection biases, they underscore the importance of a prudent approach in using knowledge from EAC case-control studies in the management of BE populations.

The currently accepted paradigm for the natural history of oesophageal adenocarcinoma (EA) is progression from a normal oesophageal stratified squamous epithelium, to inflammatory lesions, to replacement with a columnar metaplasia known as Barrett's oesophagus (BE), before transformation to adenocarcinoma (Reid et al, 2010). Overlaying an evidence-base onto this paradigm for the purposes of primary and secondary cancer prevention is not straightforward because the absolute risk of carcinogenic progression from $\mathrm{BE}$ to $\mathrm{EA}$ is low $(\sim 0.5 \%)$ (Yousef et al, 2008) and the majority ( $\sim 95 \%)$ of EA cases are diagnosed without a prior diagnosis of BE (Menke-Pluymers et al, 1992; Cooper et al, 1999; Bytzer et al, 1999; Dulai et al, 2002; Corley et al, 2002). These facts underlie the reason why we know little of risk factors for 'progression' from BE to EA, thus we may be tempted to use risk factors identified from case-control studies of EA for clinical guidance of individuals diagnosed with the precursor

*Correspondence: Dr MB Cook; E-mail: michael.cook@nih.gov

Received 14 January 2016; revised 31 August 2016; accepted 6 September 2016; published online 25 October 2016

(c) 2016 Cancer Research UK. All rights reserved 0007 - 0920/16 
metaplasia. However, we must first be confident that this knowledge is transferable by ensuring that EA diagnosed in individuals without a prior diagnosis of $\mathrm{BE}$ has a similar pathogenesis and clinical profile to incident EA following a BE diagnosis. If these two EA populations are very differentregardless of whether this is due to differences in aetiology, biology and/or selection biases-then we need to be cautious is using knowledge from EA case-control studies in the clinical management of BE. To test the assumption that EA cases are a homogeneous group, we conducted a case-case study comparing the clinical presentation, medical history and survival of EA cases with and without a prior diagnosis of BE in the SEER-Medicare database.

\section{MATERIALS AND METHODS}

Data Source. For this analysis, we used the SEER-Medicare database, which is a collaborative effort between the National Cancer Institute and the Centers for Medicare and Medicaid Services (CMS). Surveillance, Epidemiology and End ResultsMedicare comprises cancer registry data from the Surveillance, Epidemiology and End Results (SEER) Program (http:// www.seer.cancer.gov) and Medicare claims data from inpatient hospitalisations, and outpatient and physician services. The SEER data used for this analysis included 18 cancer registries and covered $\sim 28 \%$ of the US population. Medicare provides federally funded health insurance for $\sim 97 \%$ of persons aged 65 years or older in the US. Healthcare providers submit claims to Medicare using International Classification of Diseases (ICD-9) diagnosis and procedure codes, Current Procedural Terminology (CPT-4) and Healthcare Common Procedure Coding System (HCPCS) codes. SEER and Medicare data are linked biennially using a deterministic algorithm before being stripped of personal identifiers for research use.

Study population. We designed an EA case-case study-as well as a series of complementary case-control comparisons-nested within SEER-Medicare to better understand the natural history of this malignancy. The two EA case groups were created dependent on $\mathrm{BE}$ status: those with a prior diagnosis of $\mathrm{BE}$ (EA-prior $\mathrm{BE})$; and those without a prior diagnosis of $\mathrm{BE}$ (EA-no prior $\mathrm{BE})$. This provided for a direct comparison of risk factor profiles and survival patterns between the two case groups. In addition, risk factor profiles of each of these two case groups were compared with their own incidence density-matched population control group and their own incidence density-matched BE control group. These additional case-control comparisons provided additional insight into why case-case differences were observed.

EA-prior BE cases included all individuals diagnosed with EA (ICD-10: C15.0-C15.9; ICD-O: 8140-8575) in the SEER-18 registries during the period 1994-2009 with a diagnosis of $\mathrm{BE}$ (ICD-9CM code 530.2 or 530.85) at any time in their observed history but at least 6 months before the date of their EA diagnosis. Code 530.85 was introduced in 2003 and specifically identifies individuals with BE. Previous to 2003, code 530.2 (ulcer of the oesophagus) was used for diagnoses of $\mathrm{BE}$. We used all instances of code 530.85 but ceased using the less specific code of 530.2 on $1 / 1 / 2004$. This 1 year overlap between start of 530.85 and our cutoff for code 530.2 was based on tabulations of these codes by calendar year.

EA-no prior BE cases included all individuals diagnosed with EA without a prior diagnosis of BE. In addition, individuals with a first-observed diagnosis of BE within the 6-month period before their EA diagnosis were considered to be prevalent cancer cases and thus placed in this EA-no prior BE case group.

Each case group (EA-prior BE, EA-no prior BE) was separately matched to its own population control group and to its own $\mathrm{BE}$ control group using incidence density sampling with replacement of selected controls between risk sets. All controls were identified from the 5\% random sample of Medicare beneficiaries residing in the geographic regions of the SEER-18 registries (including those with cancer, except EA). Matching was based on age ( \pm 1 year), sex, race and SEER registry. Population controls were matched to cases in a 5:1 ratio and were given their matched case's cancer diagnosis date as a pseudo-diagnosis date. $\mathrm{BE}$ controls were matched to cases in a $1: 1$ ratio to each EA case group and were additionally matched on date of diagnosis (BE for controls and EA for cases; exact month and year).

Only persons enrolled in Medicare parts A and B continuously for at least 3 years before their diagnosis/pseudo-diagnosis date were eligible for inclusion to ensure an adequate period for risk factor exposure ascertainment (2 years) as well as an exposure lag period (1 year). Individuals were excluded from selection if: (i) they were enrolled in an HMO at any time in the period 3 years before their diagnosis/pseudo-diagnosis date (EA cases = 5219); (ii) data were inconsistent between the SEER and Medicare databases (name, sex, date of birth (coded) and date of death (coded)) (EA cases $=2427$ ); (iii) data on age, sex, race and SEER registry were incomplete/unknown (EA cases $=37$ ); or (iv) they were enrolled in Medicare on the basis of end-stage renal failure or disabilities (EA cases $=1795)$. Oesophageal adenocarcinoma cases identified solely from death certificates or autopsy reports were excluded from selection $(n=251)$. Oesophageal cancer cases with unknown morphology (or with non-adenocarcinoma or nonsquamous cell carcinoma histology) were also excluded from selection $(n=9538)$. Individuals diagnosed with EA before or within 6 months of their BE diagnosis date were ineligible for selection as a $\mathrm{BE}$ control.

Variables for analysis. Exposures were captured from the linked Medicare claims files for a 2-year period starting 3 years before cancer diagnosis/pseudo-diagnosis date. Exposure data from Medicare claims 1 year before cancer diagnosis/pseudo-diagnosis date served as an exposure lag window and were not used for exposure classification, due to concerns that medical claims may have been affected by a diagnosis of EA.

The following variables were only assessed in case-case comparisons (EA-prior BE vs EA-no prior BE) because these variables were used as-or related to-matching factors for the selection of controls, or they are descriptors or procedures related to cancer: age, sex, race, urban/rural status, household income (adjusted for household size), education, stage, grade and tumour size. Household income and education variables were each census tract median levels that were categorised into quintiles within registry and then aggregated quintiles across registries. Education was an amalgamated and weighted variable of the four distinct census tract education variables in SEER-Medicare. Zip code data were used when census tract data were unavailable. In addition, we assessed whether there were differences in metastases, surgery, chemotherapy and radiotherapy between these two case groups using a different exposure window for these treatment-associated variables of 1 month before 12 months post date of cancer diagnosis. We also compared survival from date of diagnosis between these two case groups.

For the case-case as well as the case-control comparisons, we assessed GERD, tobacco smoking, metabolic syndrome, obesity, hypertension, impaired fasting glucose, type II diabetes, dyslipidemia, weight loss, peptic ulcer, inflammatory bowel disease, modified Charlson comorbidity score, number of physician visits in the 2-year exposure window and Medicaid dual enrolment. Metabolic syndrome was defined as the presence of at least three of the following conditions: elevated waist circumference/central obesity (obesity served as a surrogate variable); dyslipidemia (elevated triglycerides, lowered high-density lipoprotein); hypertension; and impaired fasting 
Table 1. Descriptive characteristics of EA-prior BE cases and EA-no prior BE cases

\begin{tabular}{|c|c|c|c|}
\hline Variable & $\begin{array}{c}\text { EA-prior BE } \\
(n=662)\end{array}$ & $\begin{array}{c}\text { EA-no prior BE } \\
(n=4609)\end{array}$ & Univariate $P$-value ${ }^{a}$ \\
\hline Male, \% & 78 & 79 & 0.72 \\
\hline $\begin{array}{l}\text { Urban/rural, \% } \\
\text { Counties of metro areas of } \geqslant 1 \mathrm{M} \text { population } \\
\text { Counties in metro areas of } 250 \mathrm{~K} \text { to } 1 \mathrm{M} \text { population } \\
\text { Counties in metro areas of }<250 \mathrm{~K} \text { population } \\
\text { All urban and rural population categories combined }\end{array}$ & $\begin{array}{l}52 \\
23 \\
11 \\
14\end{array}$ & $\begin{array}{l}52 \\
20 \\
11 \\
17\end{array}$ & 0.43 \\
\hline $\begin{array}{l}\text { Household income (census tract), \% } \\
1 \text { (Lowest aggregated quintile) } \\
2 \\
3 \\
4 \\
5 \text { (Highest aggregated quintile) }\end{array}$ & $\begin{array}{l}17 \\
18 \\
20 \\
23 \\
22\end{array}$ & $\begin{array}{l}21 \\
20 \\
20 \\
20 \\
20\end{array}$ & 0.07 \\
\hline $\begin{array}{l}\text { Charlson comorbidity index, \% } \\
\text { None }(0) \\
\text { Low (1) } \\
\text { Moderate (2) } \\
\text { High }(3+)\end{array}$ & $\begin{array}{l}51 \\
27 \\
10 \\
11\end{array}$ & $\begin{array}{c}64 \\
21 \\
8 \\
6\end{array}$ & $<0.0001$ \\
\hline Number of physician visits in 2-year period, mean (s.d.) & $45(32)$ & $29(28)$ & $<0.0001$ \\
\hline Medicaid dual enrollment, \% & 5.7 & 4.4 & 0.12 \\
\hline $\begin{array}{l}\text { Cancer stage, \% } \\
\text { In situ } \\
\text { Localised } \\
\text { Regional } \\
\text { Distant } \\
\text { Unstaged }\end{array}$ & $\begin{array}{l}6 \\
52 \\
15 \\
13 \\
14\end{array}$ & $\begin{array}{l}1 \\
25 \\
29 \\
30 \\
15\end{array}$ & $<0.0001$ \\
\hline Tumour size (mm), mean (s.d.) & $31(25)$ & $50(32)$ & $<0.0001$ \\
\hline $\begin{array}{l}\text { Metastases, \% } \\
\text { None } \\
\text { Regional } \\
\text { Distant } \\
\text { Unknown }\end{array}$ & $\begin{array}{c}38 \\
7 \\
7 \\
48\end{array}$ & $\begin{array}{l}17 \\
13 \\
13 \\
56\end{array}$ & $<0.0001$ \\
\hline $\begin{array}{l}\text { Surgery, \% } \\
\text { No } \\
\text { Yes }\end{array}$ & $\begin{array}{l}50 \\
50\end{array}$ & $\begin{array}{l}73 \\
27\end{array}$ & $<0.0001$ \\
\hline Time to surgery (days), mean (s.d.) & $72(60)$ & $85(70)$ & 0.0007 \\
\hline Chemotherapy, \% & 28 & 43 & $<0.0001$ \\
\hline Radiotherapy, \% & 31 & 45 & $<0.0001$ \\
\hline $\begin{array}{l}\text { Survival time (years), mean (s.d.) } \\
\text { Overall } \\
\text { In situ } \\
\text { Localised } \\
\text { Regional } \\
\text { Distant } \\
\text { Unstaged }\end{array}$ & $\begin{array}{l}2.52(2.79) \\
4.89(3.21) \\
3.29(2.98) \\
1.66(1.96) \\
0.68(0.70) \\
1.33(2.00)\end{array}$ & $\begin{array}{l}1.45(2.03) \\
3.78(3.56) \\
2.26(2.67) \\
1.61(1.96) \\
0.72(0.99) \\
1.03(1.50)\end{array}$ & $\begin{array}{c}<0.0001 \\
0.11 \\
<0.0001 \\
0.78 \\
0.62 \\
0.17\end{array}$ \\
\hline \multicolumn{4}{|c|}{ 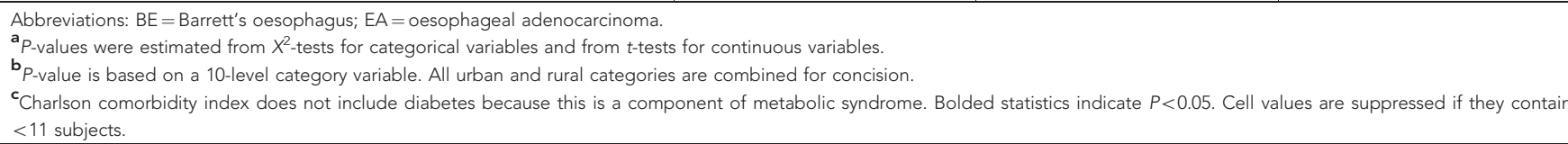 } \\
\hline
\end{tabular}


glucose/type II diabetes. In addition, an individual was considered to have a metabolic syndrome if there was a single claim for dysmetabolic syndrome X (277.7) during the specified exposure window. A modified Charlson comorbidity score was calculated based on the clinical comorbidity index developed by Charlson et al (1987), updated by Deyo et al (1992) and expanded by Klabunde et al (2007) (http://healthcaredelivery.cancer.gov/seermedicare/program/comorbidity.html?\&url=/seermedicare/program/comorbidity.html). The modified Charlson comorbidity score incorporated both inpatient and outpatient Medicare claims during the same 2-year exposure window starting 3 years before date of diagnosis/pseudo-diagnosis. Variables were excluded from the score when that variable of interest was assessed as an exposure (e.g., diabetes) or as part of an exposure (e.g., diabetes when assessing metabolic syndrome). Relevant codes for all exposures are detailed in Supplementary Table 1.

Statistical analyses. For descriptive characterisation of case groups and their matched control groups, we calculated frequencies and percentages for categorical variables, and the means and standard deviations for continuous variables. For univariate statistical case-case and case-control comparisons we used twosample $t$-tests for continuous variables and $X^{2}$ or Fisher's exact tests for categorical variables.

For case-case (EA-prior BE vs EA-no prior BE) multivariable analyses we used unconditional logistic regression to estimate odds ratios (OR) and 95\% confidence intervals (95\% CI) for associations with exposures. Multivariable models included age at diagnosis (continuous), sex, race, education, SEER registry and modified Charlson comorbidity score (categorical). Sensitivity analyses with adjustment for stage were also conducted. For case-control multivariable analyses, we used conditional logistic regression based on matched-sets (age, sex, race and SEER registry) to estimate ORs and 95\% CIs for associations of exposures. These models included the covariates education and modified Charlson comorbidity score (categorical).

For case-case (EA-prior BE vs EA-no prior BE) survival analyses, we used Cox proportional hazards regression models with T0 defined as date of cancer diagnosis. We assessed the outcomes of death from any cause and death with oesophageal cancer as the underlying cause. Individuals were right-censored at last date of follow-up, death or end of follow-up (12/31/2009), whichever occurred first. Minimally adjusted Cox proportional hazards regression models included variables deemed as known confounders (age, sex, race, education and SEER registry) and we also tested the effects of additional adjustment for clinical factors (modified Charlson comorbidity score, stage (SEER), grade (SEER), tumour size (SEER), metastases, surgery, chemotherapy and radiotherapy). Cox proportional hazards regression models were also stratified by cancer stage (localised, regional and distant) to assess potential effect-modification. We adjusted for time to surgery (amongst those receiving surgery), and restricted analyses to those not receiving surgery to further test whether this variable affected any survival advantages of having had a prior diagnosis of BE. The proportional hazards assumption was assessed through inspection of time-stratified hazards ratios. All analyses were conducted using SAS v9 (SAS Institute Inc., Cary, NC, USA). $P$-values $<0.05$ were considered to be statistically significant.

\section{RESULTS}

Demographic characteristics of EA cases with a prior diagnosis of $\mathrm{BE}$ (EA-prior $\mathrm{BE}$ ) and of EA cases without a prior diagnosis of $\mathrm{BE}$ (EA-no prior BE) are shown in Table 1. There were a total of 5271 EA cases in this study, $87 \%(n=4609)$ of which had not had a prior diagnosis of BE. Patient characteristics were mostly similar between these two case populations based on univariate comparisons with the exception that EA-prior $\mathrm{BE}$ cases had a higher number of physician visits in the 2-year period than EA-no prior BE cases.

In multivariable analyses of exposures/clinical conditions we found that risk of EA-prior BE (compared with EA-no prior BE) was positively associated with GERD, ever cigarette smoking, hypertension, dyslipidemia, weight loss, peptic ulcer and irritable bowel disease (Table 2). The OR for GERD was 11.54 (95\%CI: 9.58, 13.90), while other positively associated clinical conditions with high prevalence included hypertension $(\mathrm{OR}=1.65,95 \% \mathrm{CI}: 1.34$, 2.03 ) and dyslipidemia ( $\mathrm{OR}=1.39,95 \% \mathrm{CI}$ : 1.17, 1.67). Obesity, metabolic syndrome, impaired fasting glucose and diabetes did not differ between the case groups upon multivariable analysis. Additional adjustment for stage had negligible effects on these estimates (data not shown).

Each EA case group was compared with an incidence density matched population control group (Table 3 ) in order to provide additional insight into the origin of, and evidence for, case-case differences. Seven EA cases could not be matched to suitable

Table 2. Models of exposures in relation to EA-prior BE compared with EA-no prior BE

\begin{tabular}{|c|c|c|c|c|c|}
\hline Variable & EA-prior BE $(n=662)$ & EA-no prior BE $(n=4609)$ & Univariate $P$-value ${ }^{a}$ & \multicolumn{2}{|c|}{ Multivariable logistic regression ${ }^{\mathbf{b}}$} \\
\hline GERD, \% & 68 & 15 & $<0.0001$ & $11.54(9.58,13.90)$ & $<0.0001$ \\
\hline Metabolic syndrome, \% & 25 & 21 & 0.02 & $1.10(0.90,1.34)$ & 0.37 \\
\hline Obesity, \% & 4.2 & 4.1 & 0.90 & $0.91(0.60,1.38)$ & 0.66 \\
\hline Diabetes, \% & 30 & 27 & 0.17 & $1.02(0.85,1.23)$ & 0.80 \\
\hline Dyslipidemia, \% & 66 & 55 & $<0.0001$ & $1.39(1.17,1.67)$ & 0.0003 \\
\hline Weight loss, \% & 9.1 & 4.1 & $<0.0001$ & $2.14(1.57,2.92)$ & $<0.0001$ \\
\hline Peptic ulcer, \% & 11.9 & 3.1 & $<0.0001$ & $3.72(2.75,5.03)$ & $<0.0001$ \\
\hline
\end{tabular}




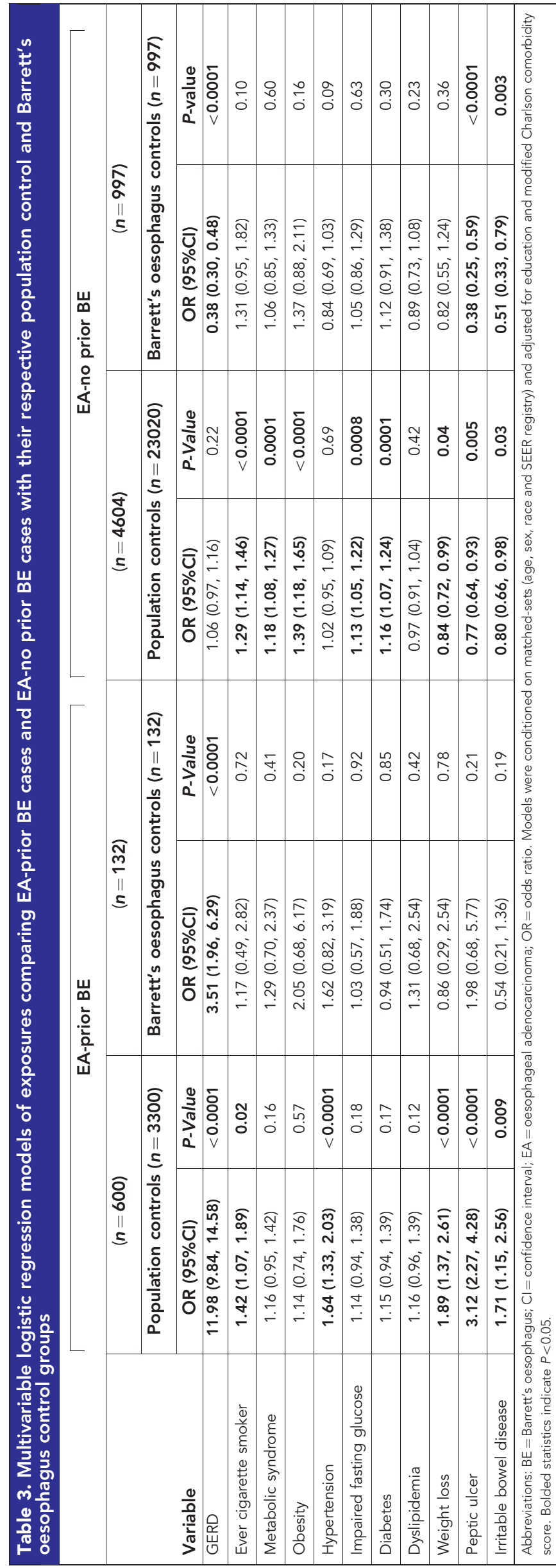

controls and were thus dropped from these conditional analyses. Notably, in contrast to the EA-prior BE case-population control comparisons shown, EA-no prior BE cases were no more likely than their population controls to have a prior diagnosis of GERD, but were more likely to have a prior diagnosis of metabolic syndrome $(\mathrm{OR}=1.18,95 \% \mathrm{CI}: 1.08,1.27)$ and be obese $(\mathrm{OR}=1.39$, $95 \%$ CI: 1.18, 1.65). EA-prior BE, meanwhile, was not associated with obesity in the multivariable model, whereas hypertension $(\mathrm{OR}=1.64,95 \% \mathrm{CI}: 1.33,2.03)$ was significantly associated. Weight loss, peptic ulcer and irritable bowel disease were distinct in that they were each positively associated with EA-prior BE but inversely associated with EA-no prior BE, when compared with population controls.

Generally similar patterns were observed when each EA case group was compared with an incidence density matched $\mathrm{BE}$ control group (Table 3), although our sample size was considerably smaller for these analyses due to a scarcity of comparable $\mathrm{BE}$ subjects when applying the matching criteria. Of note was a positive association between GERD and EA-prior $\mathrm{BE}(\mathrm{OR}=3.51$, $95 \%$ CI: 1.96, 6.29) which was inverse in the analysis of EA-no prior $\mathrm{BE}(\mathrm{OR}=0.38,95 \% \mathrm{CI}: 0.30,0.48)$. Peptic ulcer and irritable bowel syndrome remained inversely associated with EA-no prior BE, as they were when compared with population controls, but these exposures were no longer positively associated with EA-prior BE.

The univariate comparisons of tumour and treatment characteristics of the two EA case populations were starkly different (Table 1): EA-prior BE cases presented with lower stages, lower grades, smaller tumours and less metastatic disease, each relative to EA-no prior BE cases. EA-prior BE cases were also more likely to undergo surgical intervention and less likely to receive chemotherapy and radiotherapy. Overall mean survival (2.5 $v s 1.4$ years) was greater in EA-prior BE cases relative to EA-no prior BE. Upon multivariable Cox proportional hazards regression (Table 4), the survival advantage of EA-prior BE cases persisted. In models adjusted for age at diagnosis, sex, race, education and registry, the HR for overall survival was 0.53 (95\%CI: $0.48,0.58)$ and the HR for oesophageal cancer-specific mortality was $0.45(0.40,0.51)$. These differences remained evident after additional adjustment for stage, metastases and surgery $\left(\mathrm{HR}_{\text {all-cause mortality }}=0.74,95 \% \mathrm{CI}: 0.67\right.$, $0.82 ; \mathrm{HR}_{\text {oesophageal cancer-specific mortality }}=0.69,95 \% \mathrm{CI}: 0.60,0.78$ ) When stratified by stage, it was evident that these survival advantages were largely confined to localised disease. Results were not materially altered when additionally adjusted for time to surgery (amongst those who received surgery), and when restricted to those who did not receive surgery (results not shown).

\section{DISCUSSION}

In this manuscript, we showed that the risk factor profile, tumour characteristics, treatment and survival pattern for EA differed by whether or not there was a prior diagnosis of BE. Regardless of whether these differences are due to aetiology, biology and/or selection biases, we must be cautious in using knowledge from EA case-control studies in the clinical management of BE.

GERD was positively associated among the $13 \%$ of EA cases that had a prior diagnosis of BE. This may be expected given that a third or more of individuals diagnosed with BE initially present with symptomatic GERD (Menon et al, 2011; Omer et al, 2012) (personal communication: Drs Shyam Menon and Nigel Trudgill. NHS Trust Hospitals, England, UK, 3 February 2013). Less expected, perhaps, is the lack of any association of GERD with the EA-no prior BE case group, which comprised $87 \%$ of the total case population. In fact, these EA cases were substantially less likely to have GERD than their matched BE controls. Despite evidence from otherwise healthy individuals that GERD severity 
Table 4. Cox proportional hazards regression models of allcause and oesophageal cancer mortality risks for EA-prior BE cases vs EA-no prior BE cases

\begin{tabular}{|c|c|c|}
\hline \multirow[b]{2}{*}{ Variable } & \multicolumn{2}{|c|}{$\begin{array}{l}\text { Cox proportional hazards } \\
\text { regression }\end{array}$} \\
\hline & $\mathrm{HR}(95 \% \mathrm{Cl})$ & $P$-value \\
\hline \multicolumn{3}{|l|}{ All-cause mortality } \\
\hline $\begin{array}{l}\text { Overall } \\
\text { Minimally adjusted modela } \\
\text { Full-adjusted model }^{\mathbf{b}}\end{array}$ & $\begin{array}{l}0.53(0.48,0.58) \\
0.74(0.67,0.82)\end{array}$ & $\begin{array}{l}<0.0001 \\
<0.0001\end{array}$ \\
\hline $\begin{array}{l}\text { Localised stage } \\
\text { Minimally adjusted model } \\
\text { Full-adjusted model }\end{array}$ & $\begin{array}{l}0.52(0.44,0.60) \\
0.68(0.58,0.80)\end{array}$ & $\begin{array}{l}<0.0001 \\
<0.0001\end{array}$ \\
\hline $\begin{array}{l}\text { Regional stage } \\
\text { Minimally adjusted model } \\
\text { Full-adjusted model }\end{array}$ & $\begin{array}{l}0.92(0.74,1.14) \\
0.87(0.70,1.09)\end{array}$ & $\begin{array}{l}0.44 \\
0.23\end{array}$ \\
\hline $\begin{array}{l}\text { Distant stage } \\
\text { Minimally adjusted mode }\left.\right|^{\mathbf{a}} \\
\text { Full-adjusted model }\end{array}$ & $\begin{array}{l}0.91(0.73,1.14) \\
0.90(0.72,1.15)\end{array}$ & $\begin{array}{l}0.41 \\
0.43\end{array}$ \\
\hline \multicolumn{3}{|c|}{ Oesophageal cancer mortality } \\
\hline $\begin{array}{l}\text { Overall } \\
\text { Minimally adjusted model } \\
\text { Full-adjusted model }\end{array}$ & $\begin{array}{l}0.45(0.40,0.51) \\
0.69(0.60,0.78)\end{array}$ & $\begin{array}{l}<0.0001 \\
<0.0001\end{array}$ \\
\hline $\begin{array}{l}\text { Localised stage } \\
\text { Minimally adjusted model } \\
\text { Full-adjusted model }\end{array}$ & $\begin{array}{l}0.39(0.32,0.49) \\
0.58(0.46,0.73)\end{array}$ & $\begin{array}{l}<0.0001 \\
<0.0001\end{array}$ \\
\hline $\begin{array}{l}\text { Regional stage } \\
\text { Minimally adjustedmodel } \\
\text { Full-adjusted model }\end{array}$ & $\begin{array}{l}0.92(0.71,1.19) \\
0.89(0.69,1.16)\end{array}$ & $\begin{array}{l}0.54 \\
0.38\end{array}$ \\
\hline $\begin{array}{l}\text { Distant stage } \\
\text { Minimally adjusted model } \\
\text { Full-adjusted model }\end{array}$ & $\begin{array}{l}0.78(0.60,1.03) \\
0.82(0.62,1.08)\end{array}$ & $\begin{array}{l}0.08 \\
0.15\end{array}$ \\
\hline 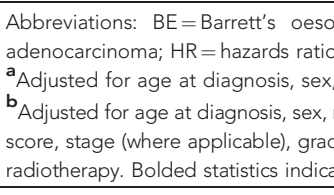 & $\begin{array}{l}\mathrm{Cl}=\text { confidence inter } \\
\text { cation and registry. } \\
\text { ation, registry, modifie } \\
\text { size, metastases, surg }\end{array}$ & $\begin{array}{l}\text { on comorbidity } \\
\text { motherapy and }\end{array}$ \\
\hline
\end{tabular}

is associated with symptoms (Bredenoord et al, 2006), it is conceivable that many EA-no prior BE cases have asymptomatic reflux. Although individuals with $\mathrm{BE}$ may have a reduced oesophageal sensitivity to GERD (Byrne et al, 2003), there is no evidence to suggest that this effect would be greater in those without a prior diagnosis of $\mathrm{BE}$ (i.e., greater length or $\mathrm{C} \& \mathrm{M}$ score of metaplasia), compared with EA-prior BE. A plausible interpretation of these findings is that a majority of individuals diagnosed with EA do not have a recent history of severe GERD exposure.

Exposures that were positively associated with EA-prior $\mathrm{BE}$ when compared with groups without BE (both EA-no prior $\mathrm{BE}$ and population controls), but null when compared with $\mathrm{BE}$ controls may be candidates for false-positive associations due to information bias (Figure 1). This idea is supported by the higher numbers of physician visits that EA-prior BE and BE control groups had-likely due to oesophageal symptoms, diagnostic workup, and surveillance of BE-relative to the groups without $B E$. Irritable bowel disease and peptic ulcer are putative falsepositive associations with EA-prior BE due to information bias given that the associations follow the above pattern; although peptic ulcer retained a positive point estimate $(O R=2)$ for $\mathrm{EA}$-prior $\mathrm{BE}$ in comparison to $\mathrm{BE}$ control groups, the inverse association with EA-no prior BE was strengthened when the comparison group changed from population controls $(\mathrm{OR}=0.8)$ to $\mathrm{BE}$ controls $(\mathrm{OR}=0.4)$. Weight loss was also a candidate false- positive risk factor of EA-prior BE, but the pattern of association was more complex-EA-no prior BE shared an inverse association with weight loss $(\mathrm{OR}=0.8)$, which is unaltered when compared with $\mathrm{BE}$ controls $(\mathrm{OR}=0.8)$, providing evidence against the idea that information bias is the cause of the attenuated association with EA-prior BE when the comparison group was switched from population controls to BE controls. The relationship between hypertension and EA-prior BE was not materially altered when compared with BE controls; the lack of nominal significance is likely due to the smaller number of subjects in the BE control model and the fact that hypertension is only weakly associated with BE (Drahos et al, 2015). Thus, hypertension may be a risk factor for EA-prior BE but not for EA-no prior BE. Although etiologic heterogeneity could be invoked, there is scant evidence for this from the other exposures assessed in this study.

We have shown that, on average, EA-prior BE cases have lower risks of all-cause and oesophageal cancer-specific death relative to EA-no prior BE cases. Two prior studies have compared all-cause mortality between EA-prior BE and EA-no prior BE case groups. The first, also based in the SEER-Medicare database, included 223 EA-prior BE cases and 2531 EA-no prior BE cases and found an HR of 0.45 (95\%CI: 0.25, 0.80) (Cooper et al, 2009). This HR is similar to what we found, even though this study used only the less specific ICD-9CM code of 530.2 for $\mathrm{BE}$ and had many fewer EA-prior BE cases. A second study, conducted in Ireland, included 52 EA-prior BE cases and 664 EA-no prior BE cases and found a similar HR of 0.44 (95\%CI: $0.30,0.64)$, although this model was only adjusted for age at diagnosis, sex and tumour grade (Bhat et al, 2015). Together, our studies provide compelling evidence for a survival benefit among EA cases diagnosed after BE. However, this survival benefit is unlikely the result of surveillance endoscopy-despite the greater proportion of EA-prior BE cases that were diagnosed with localised disease, relative to EA-no prior BE cases-because analyses with additional adjustment for time to surgery (amongst those who received surgery) and restriction to those who did not receive surgery each provided similar survival benefits. It is unlikely that survival differences can be attributed to lead time bias, since age at cancer diagnosis was higher for EA-prior BE cases. However, length bias-differences in tumour biology/ aggressiveness (including overdiagnosis) (Duffy et al, 2008)-is an alternative explanation.

Strengths of this study based on SEER-Medicare data include the large population-particularly of EA-prior BE cases-that provided for a robust assessment of the hypothesis, as well as the prospective nature of data collection through medical billing and diagnosis codes, which avoids recall and reporting biases associated with case-control status. Limitations include the ability to assess only EA cases diagnosed at 68 years and older, since SEER-Medicare is largely restricted to individuals aged 65 years and older, and our study design required time exposure ascertainment and exposure lag periods. Lastly, using medical billing for exposure ascertainment likely resulted in underascertainment for some variables, although this is likely to be non-differential for most exposures given the prospective collection of data.

In sum, our study provides evidence that EA case groups differ by whether or not they were previously diagnosed with $\mathrm{BE}$, including differences in medical history (specifically GERD), clinical presentation and survival. These findings underscore the importance of a prudent approach when attempting to reconstruct the natural history of this malignancy with utility for cancer prevention. Knowledge from total oesophageal adenocarcinoma case-series may not be directly applicable for cancer prevention efforts in BE populations. Conversely, the value gained from studying carcinogenic progression in $\mathrm{BE}$ cohorts may have limited 


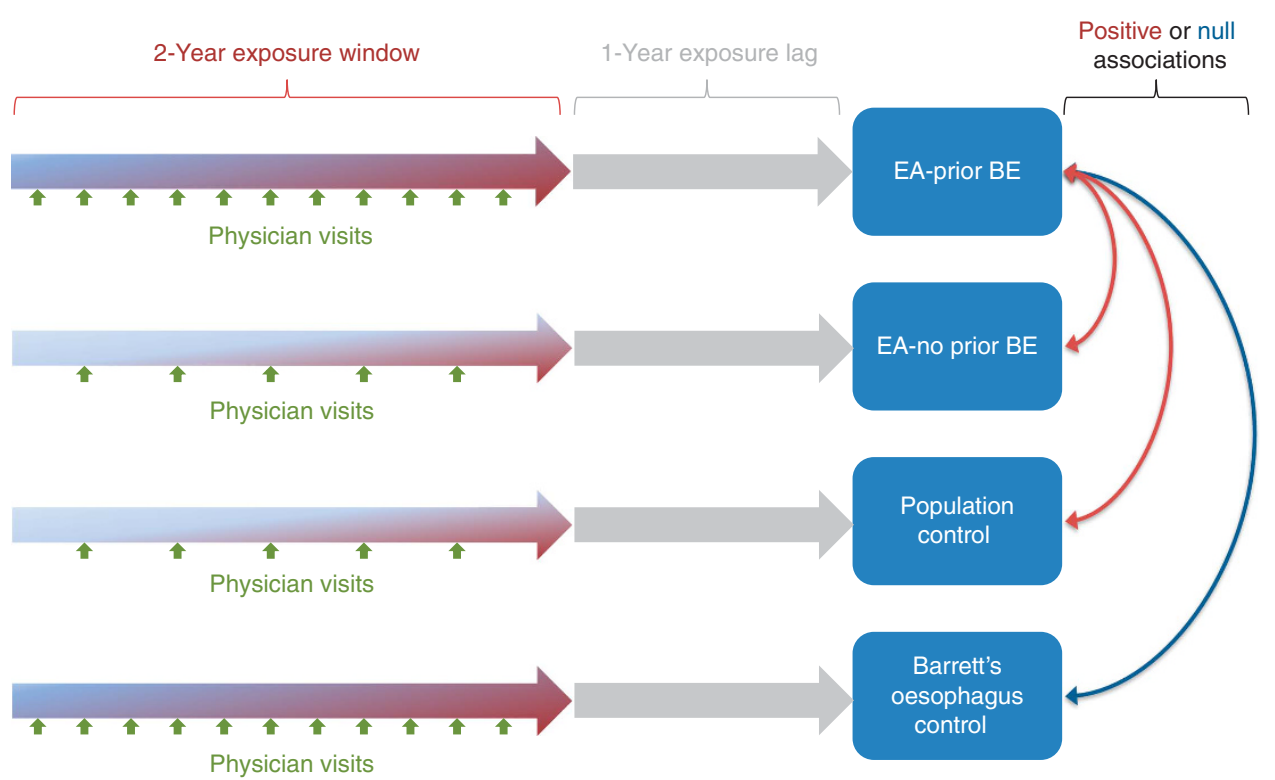

Figure 1. During the 2-year exposure window, the greater proportion of red in the horizontal arrows for EA-prior BE and BE control groups signifies the idea of greater likelihoods for exposure capture due to a greater number of physician visits for oesophageal symptoms, diagnostic workup, and surveillance of BE. False-positive associations due to exposure capture (information) bias may be indicated when associations are positive in comparisons of EA-prior BE with EA-no prior BE and Population Control groups yet null when compared with BE Controls.

translational value to larger (unidentified) populations at high-risk of developing oesophageal adenocarcinoma. Lastly, the survival benefit associated with a previous diagnosis of BE may be ascribed to residual confounding and length bias.

\section{ACKNOWLEDGEMENTS}

Intramural Program of the National Cancer Institute, National Institutes of Health, Department of Health and Human Services.

\section{CONFLICT OF INTEREST}

The authors declare no conflict of interest.

\section{AUTHOR CONTRIBUTIONS}

Study concept and design (all), acquisition of the data (MBC and WR), analysis and interpretation of the data (MBC, WR and CCA), drafting of the manuscript (MBC), critical revision of the manuscript for important intellectual content (all), statistical analysis (WR), administrative, technical or material support (SW, WR, RP, LE and MBC) and study supervision (MBC and CCA).

\section{REFERENCES}

Bhat SK, Mcmanus DT, Coleman HG, Johnston BT, Cardwell CR, Mcmenamin Ú, Bannon F, Hicks B, Kennedy G, Gavin AT, Murray LJ (2015) Oesophageal adenocarcinoma and prior diagnosis of Barrett's oesophagus: a population-based study. Gut 64: $20-25$.

Bredenoord AJ, Weusten BL, Curvers WL, Timmer R, Smout AJ (2006) Determinants of perception of heartburn and regurgitation. Gut 55: 313-318.
Byrne PJ, Mulligan ED, O’Riordan J, Keeling PW, Reynolds JV (2003) Impaired visceral sensitivity to acid reflux in patients with Barrett's esophagus. The role of esophageal motility*. Dis Esophagus 16: 199-203.

Bytzer P, Christensen PB, Damkier P, Vinding K, Seersholm N (1999) Adenocarcinoma of the esophagus and Barrett's esophagus: a populationbased study. Am J Gastroenterol 94: 86-91.

Charlson ME, Pompei P, Ales KL, Mackenzie CR (1987) A new method of classifying prognostic comorbidity in longitudinal studies: development and validation. J Chronic Dis 40: 373-383.

Cooper GS, Kou TD, Chak A (2009) Receipt of previous diagnoses and endoscopy and outcome from esophageal adenocarcinoma: a population-based study with temporal trends. Am J Gastroenterol 104: $1356-1362$.

Cooper GS, Yuan Z, Stange KC, Dennis LK, Amini SB, Rimm AA (1999) The sensitivity of Medicare claims data for case ascertainment of six common cancers. Med Care 37: 436-444.

Corley DA, Levin TR, Habel LA, Weiss NS, Buffler PA (2002) Surveillance and survival in Barrett's adenocarcinomas: a population-based study. Gastroenterology 122: 633-640.

Deyo RA, Cherkin DC, Ciol MA (1992) Adapting a clinical comorbidity index for use with ICD-9-CM administrative databases. J Clin Epidemiol 45: 613-619.

Drahos J, Ricker W, Parsons R, Pfeiffer RM, Warren JL, Cook MB (2015) Metabolic syndrome increases risk of Barrett esophagus in the absence of gastroesophageal reflux: an analysis of SEER-Medicare data. J Clin Gastroenterol 49: 282-288.

Duffy SW, Nagtegaal ID, Wallis M, Cafferty FH, Houssami N, Warwick J, Allgood PC, Kearins O, Tappenden N, O'sullivan E, Lawrence G (2008) Correcting for lead time and length bias in estimating the effect of screen detection on cancer survival. Am J Epidemiol 168: 98-104.

Dulai GS, Guha S, Kahn KL, Gornbein J, Weinstein WM (2002) Preoperative prevalence of Barrett's esophagus in esophageal adenocarcinoma: a systematic review. Gastroenterology 122: 26-33.

Klabunde CN, Legler JM, Warren JL, Baldwin LM, Schrag D (2007) A refined comorbidity measurement algorithm for claims-based studies of breast, prostate, colorectal, and lung cancer patients. Ann Epidemiol 17: 584-590.

Menke-Pluymers MB, Schoute NW, Mulder AH, Hop WC, van Blankenstein M, Tilanus HW (1992) Outcome of surgical treatment of adenocarcinoma in Barrett's oesophagus. Gut 33: 1454-1458. 
Menon S, Jayasena H, Nightingale P, Trudgill NJ (2011) Influence of age and sex on endoscopic findings of gastrooesophageal reflux disease: an endoscopy database study. Eur J Gastroenterol Hepatol 23: 389-395.

Omer ZB, Ananthakrishnan AN, Nattinger KJ, Cole EB, Lin JJ, Kong CY, Hur C (2012) Aspirin protects against Barrett's esophagus in a multivariate logistic regression analysis. Clin Gastroenterol Hepatol 10: 722-727.

Reid BJ, Li X, Galipeau PC, Vaughan TL (2010) Barrett's oesophagus and oesophageal adenocarcinoma: time for a new synthesis. Nat Rev Cancer 10: $87-101$.
Yousef F, Cardwell C, Cantwell MM, Galway K, Johnston BT, Murray L (2008) The incidence of esophageal cancer and high-grade dysplasia in Barrett's esophagus: a systematic review and meta-analysis. Am J Epidemiol 168: 237-249.

This work is published under the standard license to publish agreement. After 12 months the work will become freely available and the license terms will switch to a Creative Commons AttributionNonCommercial-Share Alike 4.0 Unported License.

Supplementary Information accompanies this paper on British Journal of Cancer website (http://www.nature.com/bjc) 